\title{
ON FIXED POINT PROPERTIES OF PLANE CONTINUA
}

\author{
BY \\ HAROLD BELL( ${ }^{(1)}$
}

The Brouwer fixed point theorem for dimension two may be stated as follows: If $f$ is a continuous complex valued function defined on a simple closed curve $D$ contained in the complex plane and $f(D)$ is contained in the topological two-cell bounded by $D, T(D)$, then every extension of $f$ to a continuous function defined on $T(D)$ fixes a point in $T(D)$, i.e., if $g$ is such an extension, then there is a point $x$ in $T(D)$ for which $x=g(x)$.

If $A$ is a bounded subset of the plane, then $T(A)$ shall denote the smallest bounded subset of the plane that contains $A$ and does not separate the plane. $T(A)$ could be more precisely defined as the complement of the unbounded component of the complement of $A$.

One might ask if it is necessary that $D$ be a simple closed curve for the above statement of the Brouwer Fixed Point Theorem to hold. That is, will the theorem still hold if $D$ is an arbitrary compact connected subset of the plane? The following partial answer is the main purpose of this paper.

THEOREM 1. Let $f: M \rightarrow T(M)$ be a continuous function defined on a plane continuum, $M$. Either every extension of $f$ to a continuous function defined on $T(M)$ fixes a point or there is an indecomposable continuum $Q$ contained in $M$ such that $Q=f(Q)$.

Recall that a continuum is a compact connected Hausdorff space and that a continuum is indecomposable if it cannot be written as the union of two of its proper subcontinua. Examples of indecomposable continua can be found in [4]. Of particular interest here is the indecomposable continuum known as the "lakes of Wada." The continuum $Q$ of Theorem 1 will bear considerable resemblance to the "lakes of Wada."

Once Theorem 1 has been established the following holds:

THEOREM 2. Let $M$ be a plane continuum and $f$ be a continuous function defined on $T(M)$ for which $f(M) \subset T(M)$. Then there is an indecomposable continuum $Q \subset T(M)$ such that $Q=f(Q)$ and if $A$ is any subcontinuum of $T(Q)$ for which $f(A) \subset T(A)$, then $T(Q)=T(A)$.

Proof. The Hausdorff maximality principle assures us of the existence of a

Received by the editors February 5, 1965 and, in revised form, February 7, 1966.

(1) The author wishes to express his sincere gratitude to G. S. Young and L. B. Trehbig of Tulane University for their help and patience in the writing of this dissertation. 
maximal chain in the set of $T(X)$ where $X$ is a subcontinuum of $T(M)$ for which $f(X) \subset T(X)$. If $C$ is the intersection of such a chain and $Q$ is the boundary of $C$ then $f(Q) \subset C=T(C)$.

Notation. All sets will be assumed to be subsets of the plane unless otherwise indicated. The topological closure of a set $A$ will be denoted by $A^{*} . C(A)$ will denote the convex hull of a set $A$.

A slight generalization of Theorem 1 that allows us to deal with not necessarily continuous functions will be easier to prove than Theorem 1 itself. A function defined on a set $A$ will be said to be locally bounded at a point $x \in A^{*}$ if there is an open set $U$ containing $x$ for which $f(U \cap A)$ is bounded. $f$ is said to be locally bounded if it is locally bounded at each point of $A^{*}$. If $f$ is a locally bounded function defined on a set $A$ and $a \in A^{*}$ then $f_{c}(a)$ is defined by

$$
f_{c}(a)=\bigcap\left\{[C(f(U \cap A))]^{*}: a \in U \text { and } U \text { is open }\right\} .
$$

If $B \subset A^{*}$ then $f_{c}(B)$ shall be used to denote $\bigcup\left\{f_{c}(x): x \in B\right\}$.

Lemma 1. Let $f$ be a locally bounded function defined on a set $A$. Then

(1) $f$ is continuous at a point $x$ of $A$ if and only if $f_{c}(x)=\{f(x)\}$.

(2) If $g$ is the restriction of $f$ to some subset $B$ of $A$, then $g_{c}(x) \subset f_{c}(x)$ for each $x \in B^{*}$.

(3) $f_{c}$ is an upper semicontinuous multivalued function, i.e., if $x_{n} \rightarrow x$ and $y_{n} \rightarrow y$ where each $y_{n} \in f_{c}\left(x_{n}\right)$, then $y \in f_{c}(x)$.

The proof of Lemma 1 is straightforward and is left to the reader.

The following extension of Theorem 1 will be proved.

THEOREM 1'. Let $M$ be a continuum and let $g$ be a locally bounded function defined on the plane for which $g(x) \in T(M)$ if $x$ is not in the interior of $T(M)$. If $g$ is continuous at each point of $M$, then either $g_{c}$ has a fixed point in $T(M)$ or there is an indecomposable continuum $Q \subset M$ such that $Q=g(Q)$.

A fixed point of a multivalued function $F$ is a point $x$ for which $x \in F(x)$. Notice that if $f$ is a function defined on $T(M), f(M) \subset T(M), f$ continuous on $\mathrm{M}$, and $g$ is defined on the entire plane by letting $g(x)=f(m)$ for some $m \in T(M)$ for which $|x-m|=\inf \{|x-y|: y \in T(M)\}$, then $g$ satisfies the conditions of Theorem $1^{\prime}$.

Much work has been done concerning fixed point theorems for continuous multivalued functions. For example, in [2] Kakutani proved that if $M$ is a compact convex subset of Euclidean $n$-space and $F$ is a continuous multivalued function defined on $M$ and each $F(x)$ is a compact convex subset of $M$, then $F$ has a fixed point. In [1] Eilenberg and Montgomery extended the above result of Kakutani by proving that if $F$ is an upper semicontinuous multivalued function defined on a compact convex subset of Euclidean $n$-space and each $F(x)$ is an acyclic continuum in $n$-space and $F(x) \subset M$ for each $x$ in the boundary of $M$, then $F$ has a fixed point. Since for every simple closed curve in the plane, $D$, there is a homeomorphism of 
the plane onto itself $h$ for which $h[T(D)]$ is the unit disk, one can conclude the following from the theorem of Eilenberg and Montgomery:

LEMMA 2. Let $f$ be a bounded function defined on $T(D)$ for some simple closed curve $D$ in the plane. If $f_{c}(D) \subset T(D)$ then $f_{c}$ has a fixed point.

LEMMA 3. If $U$ is an open subset of the plane that contains a plane continuum $M=T(M)$ then there is a simple closed curve $D$ such that $T(M)$ is contained in the interior of $T(D)$ and $T(D)$ is contained in $U$.

It will become necessary to construct simple closed curves satisfying the conclusion of Lemma 3 later on in this paper. The proof of Lemma 3 is deferred until then. The following is a generalization of Lemma 2. The proof will not depend on Lemma 2.

LEMMA 4. Let $D$ be a simple closed curve and let $f$ be a bounded function defined on $T(D)$ for which $f(D) \subset T(D)$. If for each point $d \in D$ there is an open set $U$ containing $d$ such that for any two points $e$ and $h$ contained in $U \cap D$ there is an arc $A$, contained in $T(D)$, joining $f(e)$ to $f(h)$, for which $T(A \cup[f(e), f(h)]) \cap U=\varnothing$, then $f_{c}$ has a fixed point.

Proof. Suppose $f_{c}$ has no fixed point. Then there is a topological simplicial decomposition of $T(D)$ into two-cells, $K$, for which the following holds:

(a) $C(f(B)) \cap B=\varnothing$ for $B \in K$.

(b) If $L$ is a side of some $B(L) \in K$ with endpoints $a$ and $b$ and $L \subset D$, then there is an arc $A(L)$ joining $f(a)$ to $f(b)$, contained in $T(D)$, such that $T(A(L) \cup[f(a)$, $f(b)]) \cap B(L)=\varnothing$.

The compactness of $T(D)$ together with the hypothesis of the theorem assures the existence of $K$. Let $r$ be a linear extension of the restriction of $f$ to the vertices of $K$ to all of $T(D)$. Let $S$ be the collection of sides of two-cells of $K$ that are contained in $D$. For each $L \in S$ let $B(L)$ denote the $B$ in $K$ that contains $L$ and let $D(L)$ be a simple closed curve for which the interior of $T[D(L)]$ contains $T[A(L) \cup r(L)]$ and $T[D(L)] \cap B(L)=\varnothing$.

The existence of the $D(L)$ follows from Lemma 3.

Next $\{P(L): L \in S\}$ may be chosen so that

(1) Each $P(L)$ is an arc, contained in $B(L)$, that intersects $L$ exactly at the common endpoints of $L$ and $P(L)$.

(2) $r[L \cup P(L)] \subset D(L)$.

(3) No two distinct $T[L \cup D(L)]$ intersect except possibly at an endpoint of one of the $L$ 's.

Now, with the aid of the Tietze extension theorem, a continuous function $k$ may be defined on $T(D)$ with the properties

(i) $k(x)=r(x)$ if $x \notin \bigcup\{T[L \cup P(L)]: L \in S\}$.

(ii) $k(x)=r(x)$ if $x \in \bigcup\{P(L): L \in S\}$. 
(iii) $k(L)=A(L)$ if $L \in S$.

(iv) $k(T[L \cup P(L)]) \subset D(L)$ for $L \in S$.

$k$ is then a continuous function defined on $T(D)$ for which $k(D) \subset T(D)$ and $k$ has no fixed points. This contradicts the Brouwer fixed point theorem.

A set $L$ will be called a ray if there is a homeomorphism of the set of nonnegative real numbers, $h$, onto $L$ such that $\lim _{x \rightarrow \infty}|h(x)|=\infty$. $h(0)$ will be called the endpoint of the ray.

Lemma 5. Let $M$ be a plane continuum and let $g$ be a locally bounded function defined on the entire plane that is continuous at each point of $M$ and is such that $g(x) \in T(M)$ if $x$ is not in the interior of $T(M)$. Let $B=\{x: x \notin T(M)$ and $x \in$ $\left.T\left[M \cup g_{c}(x)\right]\right\}$. Then if $D$ is a simple closed curve for which $M \subset T(D)$, either $g_{c}$ has a fixed point in $T(D)$ or $D$ intersects $B$.

Proof. Suppose that $D$ does intersect $B$. Let $f$ be the restriction of $g$ to $T(D)$. By Lemma $1 f_{c}(x) \subset g_{c}(x)$ for $x \in T(D)$. According to Lemma 4 , it is sufficient to show that for each $d \in D$ there is an open set $U$ containing $d$ such that if $x, y \in D \cap U$ then there is an arc $A$ contained in $T(D)$ joining $f(x)$ to $f(y)$ such that

$$
T(A \cup[f(x), f(y)])
$$

does not intersect $U$. If $d \in M$ the existence of $U$ follows from the fact that $f$ is continuous at $d$ and $g(x) \in T(M) \subset T(D)$. If $d \notin M$ since $d \notin B$ then there is a ray $L$ with endpoint $d$ that does not intersect $T\left[M \cup f_{c}(d)\right]$. Therefore there exist open sets $U$ and $V$ such that $f_{c}(d) \subset V, d \in U, C[f(U)] \subset V, U$ is connected, and

$$
V^{*} \cap L=V^{*} \cap U^{*}=U^{*} \cap T(M)=\varnothing .
$$

According to Lemma 3 there is a simple closed curve $J$ such that $M \subset T(J)$ and $T(J) \cap\left(L \cup U^{*}\right)=\varnothing$. For $x, y \in U \cap D$ let $A(x, y)$ be any arc contained in $T(D) \cap T(J)$ that joins $f(x)$ to $f(y)$. Such arcs exist because

$$
\{f(x), f(y)\} \subset T(M) \subset T(D) \cap T(J) .
$$

A sufficient condition for a continuum to be indecomposable is

LEMMA 6. Let $h$ be a homeomorphism of the set of real numbers $R$ into the plane for which $\lim _{x \rightarrow-\infty}|h(x)|=\infty$ and $h([0, \infty))$ is bounded. Let $X=[h(R)]^{*}-h(R)$. If for each $x \in X$ and each $e>0$ there is a component $Q$ of $\{z:|z-x|=e\}-X$ and a real number $r$ for which $h(t) \in T(Q \cup X)$ for $t>r$, then $X$ is indecomposable.

Proof. Suppose that $X$ can be written as the union of two of its proper subcontinua $A$ and $B$. Let $U$ be a connected open set that intersects $A$ but whose closure does not intersect $B$. Let $v$ be a real number for which $h(v) \in U$. Let $b \in B-A$ and choose $e>0$ so that $\{z:|z-b|=e\} \cap(U \cup h((-\infty, v]))=\varnothing$. Now there is a component $Q$ of $\{z:|z-b|=e\}-X$ and a real number $r$ for which $h(t) \in T(Q \cup X)$ 
for $t>r$. Since both endpoints of $Q$ are in $B, h(t) \in T(Q \cup B)$ for $t>r$. Therefore $X \subset[h((r, \infty))]^{*} \subset T(Q \cup B)$. This is impossible since the unbounded connected set $U \cup h[(-\infty, v]]$ does not intersect $Q \cup B$.

Lemma 7. Let $M$ be a continuum and let $N$ be a compact set. Let $x \in T(M \cap N)-$ $T(M)$. Then there is a component of $N-M, K$, such that $x \in T(K \cup M)$.

Proof. Suppose not. Then let $P$ be a minimal compact subset of $N$ with the property that $x \in T(P \cup M)$. Assuming $P-M$ is not connected we may write $P-M=U \cup V$ where $U$ and $V$ are mutually disjoint nonempty sets that are closed in $P-M$. A simple closed curve $D$ may then be found such that $D$ is the union of two arcs $A_{1}$ and $A_{2}$ where $A_{1} \cap A_{2}=\left\{x_{0}, y_{0}\right\}, x_{0} \in T(M \cup P), y_{0} \notin T(M \cup P)$, and $A_{1} \cap T(U \cup M)=A_{2} \cap T(V \cup M)=M \cap T(D)=\varnothing$. Therefore $(U \cup V) \cap T(D)$ is a closed set that separates $x_{0}$ from $y_{0}$ in $T(D)$. This is impossible since $T(D)$ is unicoherent and locally connected and neither $U$ nor $V$ can intersect both $A_{1}$ and $A_{2}$.

Approximating nonseparating plane continua with two-cells. It is well known among analytic topologists that every nonseparating plane continuum is the intersection of a descending chain of topological two-cells. Since this paper requires the use of a particular descending chain of two-cells, a proof of this and some other facts will be presented in this section.

Let $M$ be a fixed plane continuum. For each natural number $n$ let $S_{n}$ be the collection of square two-cells consisting of points of the form $(a, b)$ where $k / 2^{n} \leqq a \leqq(k+1) / 2^{n}, j / 2^{n} \leqq b \leqq(j+1) / 2^{n}$ with $k$ and $j$ integers. Let $F_{n}$ be the collection of the boundaries of the two-cells in $S_{n}$. Let $K_{n}$ be the collection of open arcs that are components of some $F-T(M)$, where $F \in F_{n}, F \notin T(M)$, and $F \cap T(M) \neq \varnothing$. For each natural number $n$ let $A_{n}=T\left[\bigcup\left\{S \in S_{n}: S \cap M \neq \varnothing\right\}\right]$. Let $D_{n}$ be the boundary of $A_{n}$.

LEMMA 8. Each $D_{n}$ is a simple closed curve.

Proof. Let $n$ be a fixed natural number. It is clear from the definition of $D_{n}$ that $D_{n}$ contains a simple closed curve $D$. Since $M$ is contained in the interior of $A_{n}$, $D \cap M=\varnothing$. Consequently either $M \subset T(D)$ or $M \cap T(D)=\varnothing$. Now if $I$ is a side of some $S \in S_{n}$ that is contained in $D$, then $I$ is contained in exactly two members of $S_{n}, S^{\prime}$ and $S^{\prime \prime}$, where $S^{\prime \prime} \subset T(D)$ and $S^{\prime} \notin A_{n}$. Since $S^{\prime} \notin A_{n}, S^{\prime \prime} \subset A_{n}$, and $S^{\prime} \cup S^{\prime \prime}$ is connected, $S^{\prime \prime}$ must intersect $M$. Therefore $M \subset T(D)$. Therefore

$$
A_{n}=T\left[\bigcup\left\{S \in S_{n}: S \cap M=\varnothing\right\}\right] \subset T(D) \subset T\left(A_{n}\right)=A_{n} .
$$

Therefore $T(D)=A_{n}$ and $D_{n}$ is the boundary of $T(D)$ which is of course $D$.

LEMMA 9. $T(M)=\bigcap_{n=1}^{\infty} T\left(D_{n}\right)$ and $T\left(D_{n}\right) \supset T\left(D_{n+1}\right)$ for each natural number $n$.

Proof. Clearly $T(M) \subset \bigcap_{n=1}^{\infty} T\left(D_{n}\right)$. If $x \notin T(M)$ there is a ray with endpoint $x$ that does not intersect $T(M)$. Choose $n^{\prime}$ sufficiently large so that no $S \in S_{n}^{\prime}$ can 
intersect both $M$ and the ray. The fact that $T\left(D_{n}\right) \supset T\left(D_{n+1}\right)$ follows from the definition of the $D_{n}$.

LEMMA 10. Let $\mathbf{K}^{\prime} \subset \bigcup_{n=1}^{\infty} K_{n}$ and let $j$ be a fixed natural number, then

(i) $T\left(\bigcup\left\{K: K \in \mathbf{K}^{\prime}\right\} \cup M\right)=\bigcap_{n=j}^{\infty} T\left(\bigcup\left\{K: K \in \mathbf{K}^{\prime}\right\} \cup D_{n}\right)$.

(ii) $\bigcup\left\{K: K \in \mathbf{K}^{\prime}\right\} \cup M$ is closed.

(iii) The boundary of $T\left(\cup\left\{K: K \in \mathbf{K}^{\prime}\right\} \cup D_{j}\right)$ is a simple closed curve.

Proof. (i) is straightforward. (ii) and (iii) follow from the fact that there are at most a finite number of $K \in \bigcup_{n=1}^{\infty} K_{n}$ that have diameter greater than any fixed positive number. Consequently $A_{j}$ contains all but a finite number of the $K \in \mathbf{K}^{\prime}$. The lemma now follows by a simple finite induction procedure.

Permanent notation for the remainder of the proof of Theorem 1.

(i) Let $M$ be a plane continuum and let $g$ be a locally bounded function defined on the plane that is continuous at each point of $M$ and is such that $g(x) \in T(M)$ if $x$ is not in the interior of $T(M)$. It will be assumed that $g_{c}$ has no fixed points in $T(M)$.

(ii) A subcontinuum $Q$ of $M$ is chosen as follows: If the boundary of $M$ contains a proper subcontinuum $W$ for which $g(W) \subset W$, then let $Q$ be a minimal such. If not let $Q$ be the boundary of $T(M)$. Let $f$ be an extension of the restriction of $g$ to $T(Q)$ such that $f$ is continuous at each point of $Q$ and $f(x) \in T(Q)$ if $x$ is not in the interior of $Q$.

(iii) For each natural number $n$ define $S_{n}$ and $F_{n}$ as before. Let $K_{n}$ be the collection of open arcs that may be written as $F-T(Q)$ for some $F \in F_{n}$ for which

$$
F \cap T(Q) \neq \varnothing, F \notin T(Q) .
$$

Let $\mathbf{K}^{\prime}=\bigcup_{n=1}^{\infty} K_{n}$. Let $A_{n}=T\left(\bigcup\left\{S \in S_{n}: S \cap Q \neq \varnothing\right\}\right)$ and let $D_{n}$ be the boundary of $A_{n}$.

(iv) Let $B=\left\{x: x \notin T(Q)\right.$ and $x \in T\left(Q \cup f_{c}(x)\right\}$.

(v) Since the set of fixed points of $f_{c}$ is closed and according to Lemma 9, $T(Q)=\bigcap_{n=1}^{\infty} A_{n}$, there is a natural number $n_{0}$ for which $A_{n_{0}}$ contains no fixed points of $f_{c}$.

(vi) Let $\mathbf{K}=\bigcup_{n=n_{0}}^{\infty} K_{n}, \mathbf{K}^{\prime}=\{K: K \in \mathbf{K}$ and $K \cap B=\varnothing\}$, and $K^{\prime}=\bigcup\left\{K: K \in \mathbf{K}^{\prime}\right\}$.

(vii) Let $Y=T\left(Q \cup K^{\prime}\right)$.

(viii) For each natural number $n \geqq n_{0}$ let $J_{n}$ be the boundary of $T\left(Y \cup D_{n}\right)$ and let $H_{n}=J_{n} \cap Y$.

(ix) A point $x$ of a continuum $M$ will be called an accessible point of $M$ if there is a ray with endpoint $x$ that intersects $M$ only at the point $x$.

It has been proved that:

(1) Each $D_{n}$ and each $J_{n}$ is a simple closed curve.

(2) $Y=\bigcap_{n=n_{0}}^{\infty} T\left(J_{n}\right)$.

(3) $T\left(D_{n}\right) \supset T\left(D_{n+1}\right)$ for each natural number $n$.

(4) If $D$ is a simple closed curve for which $Q \subset T(D) \subset T\left(D_{n_{0}}\right)$, then $D$ intersects $B$. 
LEMMA 11. (i) If $n \geqq n_{0}$ then $H_{n} \subset H_{n+1}$.

(ii) If $L$ is a ray that intersects $T(Q)$ only at its endpoint, $x$, then there is an open arc having $x$ as one endpoint that is contained in both $L$ and the interior of $Y$. Consequently no accessible point of $Y$ is in $T(Q)$.

(iii) $y$ is an accessible point of $Y$ if and only if there is an $n \geqq n_{0}$ for which $y \in H_{n}$.

(iv) $K^{\prime}$ contains the set of accessible points of $Y$.

Proof. (i) $H_{n} \subset Y \subset T\left(Y \cup D_{n+1}\right)$. Since $H_{n}$ is contained in the boundary of $T\left(Y \cup D_{n}\right)$ and $T\left(Y \cup D_{n}\right) \supset T\left(Y \cup D_{n+1}\right), H_{n}$ is on the boundary of $T\left(Y \cup D_{n+1}\right)$ which by definition is $J_{n+1}$.

(ii) Let $L$ be a ray with endpoint $x$ that intersects $T(Q)$ only at $x$. Since $f$ is continuous at $x$ and $f(x) \in T(Q)$ there is a natural number $n \geqq n_{0}$ for which the convex hull of $f\left(\bigcup\left\{S \in S_{n}: x \in S\right\}\right)$ intersects neither $L$ nor $\bigcup\left\{S \in S_{n}: x \in S\right\}$. Let $W$ be the boundary of $\bigcup\left\{S \in S_{n}: x \in S\right\}$. Let $p$ be a point distinct from $x$ that is in the component of $L-W$ that contains $x$. Clearly $p \in T(Q \cup W)$. Therefore, by Lemma 7, $p \in T(Q \cup N)$ for some component $N$ of $W-T(Q)$. Now $N$ is contained in the union of those $K$ in $\mathbf{K}$ that are contained in $T(W)$ and intersect $N$. Now if $K \in \mathbf{K}$ and $K$ intersects $N$ then $f_{c}(K)$ does not intersect the connected unbounded set $K \cup N \cup L$. Therefore no $K$ in $\mathbf{K}$ that intersects $N$ can intersect $B . N$ is therefore contained in $Y$ and we can conclude that the component of $L-W \cup\{x\}$ that contains $p$ is contained in the interior of $T(Q \cup N) \subset Y$.

(iii) Let $y \in H_{n}$ for some $n \geqq n_{0}$. Since $y$ is an accessible point of $T\left(J_{n}\right), y \in Y$, and $Y \subset T\left(J_{n}\right), y$ is an accessible point of $Y$. Conversely let $y_{0}$ be an accessible point of $Y$. Let $L$ be a ray with endpoint $y_{0}$ that intersects $Y$ only at $y_{0}$. Since $y_{0} \notin T(Q)$ there is an $n \geqq n_{0}$ for which $T\left(D_{n}\right)$ does not intersect $L$. Clearly $y_{0} \in Y \cap J_{n}=H_{n}$.

(iv) follows from (iii).

Let $A(Y)$ denote the set of accessible points of $Y$.

LEMMA 12. (i) $A(Y)$ contains no simple closed curve.

(ii) $A(Y)$ is open relative to $[A(Y)]^{*}$. Furthermore each component of $A(Y)$ is homeomorphic to the set of real numbers.

(iii) $[A(Y)]^{*}-A(Y) \subset Q$.

Proof. (i) If $A(Y)$ contained a simple closed curve $D$ then we would have $Q \subset T(D) \subset T\left(D_{n_{0}}\right)$ and $B \cap D=\varnothing$. This contradicts the conclusion of Lemma 5 .

(ii) Let $y \in A(Y)$ and let $W=\bigcup\left\{K: K \in \mathbf{K}^{\prime}\right.$ and $\left.y \in K\right\}$. Let $V$ be the polygonal arc that is the intersection of the boundary of $T(W \cup Q)$ and $W$. Clearly $y \in V$. Since $y \notin T(Q)$, an open set $U$ can be found, that contains $y$, and that intersects only those $K \in \mathbf{K}$ that contain $y$. Furthermore $U$ can be chosen so that both $U \cap V$ and $U \cap\left[R^{2}-T(Q \cup V)\right]$ are connected. It is easy to see that $U \cap V \subset A(Y)$. $A(Y)$ is therefore a 1-manifold that is not a simple closed curve. The conclusion now follows.

(iii) It will be shown that each point of $[A(Y)]^{*}$ that is not in $Q$ is in $A(Y)$. 
Let $y \in[A(Y)]^{*}-Q$. Let $U^{\prime}$ be an open set containing $y$ whose closure does not intersect $T(Q)$. Since $U^{\prime}$ intersects only finitely many $K \in \mathbf{K}$ and

$$
A(Y) \subset K^{\prime}=\bigcup\left\{K: K \in \mathbf{K}^{\prime}\right\}
$$

$y$ is in some $K \in \mathbf{K}^{\prime}$. Let $U$ and $V$ be defined as in the proof of (ii). Since $U$ intersects $A(Y), y \in U \cap V \subset A(Y)$.

Some final definitions. Let $y_{0}$ be a fixed accessible point of $Y$ and let $L\left(y_{0}\right)$ be one of the components of $A(Y)-\left\{y_{0}\right\}$ that has $y_{0}$ as an endpoint. Let $L^{\prime}$ be a ray that intersects $Y$ only at $y_{0}$ and has $y_{0}$ as an endpoint. Let $L=L^{\prime} \cup L\left(y_{0}\right)$ and let $h$ be a homeomorphism of the set of real numbers onto $L$ for which $h((-\infty, 0])=L^{\prime}$.

LEMMA 13. (i) $L^{*}-L$ is a subcontinuum of $Q$.

(ii) $f\left(L^{*}-L\right) \subset L^{*}-L$. Hence $L^{*}-L=Q=f\left(L^{*}-L\right)$.

(iii) If $A$ is a closed arc that intersects $Q$ only at the endpoints of $A$ then $h^{-1}(A)$ is bounded.

(iv) $Q$ is indecomposable.

Proof. (i) It follows from Lemma 12 that $L^{*}-L$ is compact. A proof that $L^{*}-L$ is connected can be found in [4].

(ii) Let $x \in L^{*}-L$ and suppose that $f(x) \notin L^{*}-L$. Since $f$ is continuous at $x$ and $f(x) \notin L$, a natural number $n \geqq n_{0}$ can be found for which $f_{c}\left(\bigcup\left\{S \in S_{n}: x \in S\right\}\right)$ does not intersect $L$ or $\bigcup\left\{S \in S_{n}: x \in S\right\}$. Since $x \in L^{*}$ the interior of

$$
\bigcup\left\{S \in S_{n}: x \in S\right\}
$$

contains some point $z \in L\left(y_{0}\right)$. Let $W$ be the boundary of $\bigcup\left\{S \in S_{n}: x \in S\right\}$ and let $\Sigma$ be some component of $W-T(Q)$ for which $z \in T(\Sigma \cup Q)$. Since $\Sigma \cup L$ is a connected unbounded set that does not intersect $f_{c}[T(W)]$, no $K \in \mathbf{K}$ that is contained in $W$ and intersects $\Sigma$ can intersect $B . \Sigma$ is therefore contained in $K^{\prime}$. Therefore $z$ is in the interior of $T(\Sigma \cup Q) \subset T\left(K^{\prime} \cup Q\right)=Y$. This is impossible since $z$ is a boundary point of $Y$.

(iii) $h^{-1}(A)$ is clearly bounded from below. If $h^{-1}(A)$ is not bounded from above then $A \cap L\left(y_{0}\right)$ has a limit point $z \in Q$. Now since $z \in A$ there is a ray contained in $L \cup A$ with endpoint $z$. This contradicts part (ii) of Lemma 11 .

(iv) According to Lemma 6 it is sufficient to show that if $e>0$ and $x \in Q$ and if $D=\{z:|z-x|=e\}$ then there is a component $D^{\prime}$ of $D-T(Q)$ and a real number $r$ for which $h(t) \in T\left(Q \cup D^{\prime}\right)$ for any $t>r$. Let $\left\{x_{i}\right\}$ and $\left\{y_{i}\right\}$ be sequences of real numbers defined as follows: Let $x_{1}$ be the smallest real number contained in $h^{-1}(D)$. If $x_{i}$ has been defined let $y_{i}$ be the largest real number for which $h\left(x_{i}\right)$ is in the same component of $D-T(Q)$ as $h\left(y_{i}\right)$. If $y_{i}$ has been defined, let $x_{i+1}$ be the smallest real number in $h^{-1}(D)$ that is greater than $y_{i}$. Let $h^{\prime}$ be a homeomorphism of the set of real numbers into the plane defined so that $h^{\prime}(x)=h(x)$ if $x \notin \bigcup\left\{\left[x_{i}, y_{i}\right]: i=1,2, \ldots\right\}$, 
$h^{\prime}\left(\left[x_{i}, y_{i}\right]\right)$ is contained in the component of $D-T(Q)$ that contains $x_{i}$ and $y_{i}$. (If $x_{i}=y_{i}$ we let $\left[x_{i}, y_{i}\right]$ denote $\left\{x_{i}\right\}$.) Let $L^{\prime \prime}$ be the image of $h^{\prime}$. We may now apply almost the same proof as used in Lemma 13, part (ii), to show that $L^{\prime \prime *}-L^{\prime \prime}$ is a subcontinuum of $Q$ for which $f\left(L^{\prime \prime *}-L^{\prime \prime}\right) \subset L^{\prime \prime *}-L^{\prime \prime}$. Hence $Q=L^{\prime \prime *}-L^{\prime \prime}$. $r$ may now be chosen to be any real number for which $\left|h^{\prime}(r)-x\right|<e . D^{\prime}$ may be chosen to be any component of $D-T(Q)$ for which $h(r) \in T\left(D^{\prime} \cup Q\right)$.

Some remarks concerning the continuum $Q$. The following is an outline of an alternative, more intuitive, proof of Theorem 1. It was not used because it is technically messy. It is inserted here for intuitive purposes only. No proofs will be given.

Let $M$ be a nonseparating plane continuum. For $x \notin M$ let

$$
K_{x}=\{z \in M:|z-x|=\inf \{|m-x|: m \in M\}\} .
$$

Let $E(M)$ be the set of points $x$ for which $K_{x}$ has at least two distinct points. $E(M)$ may be partially ordered as follows: If $x, y \in E(M)$ then $x \leqq y$ if there exist $y_{0}, y_{1} \in K_{y}$ such that $x \in T\left(M \cup\left[y_{0}, y\right] \cup\left[y, y_{1}\right]\right)$. For $e \in E$ let $L_{e}$ denote $\{z \in E: e \leqq z\}$. It can be shown that $L_{e}$ is a ray with endpoint $e$ and furthermore every maximal ordered subset of $E(M)$ is homeomorphic to the set of real numbers. Furthermore Lemma 6 asserts that if $L$ is a maximal ordered subset of $E(M)$ and $L^{*}$ contains the boundary of $M$ then the boundary of $M$ is indecomposable.

Now one may show that if $f$ is a continuous function defined on $M$ and the image of the boundary of $M$ under $f$ is contained in $M$ and $f$ has no fixed points then there is a maximal ordered subset $L$ of $E(M)$ and a $z \in L$ such that if $x \in L$ and $x \leqq z$, then there are $x_{0}, x_{1} \in K_{x}$ such that if $A$ is any arc joining $f\left(x_{0}\right)$ to $f\left(x_{1}\right)$ that does not intersect $L_{x}$ then $x \in T\left(A \cup\left[f\left(x_{0}\right), f\left(x_{1}\right)\right]\right)$. In particular $\left[f\left(x_{0}\right), f\left(x_{1}\right)\right] \cap L_{x} \neq \varnothing$. Consequently the boundary of $M$ contains a subcontinuum $N=L^{*}-L$ for which $f(N) \subset N$. If $Q$ is chosen to be a minimal such and the above argument is repeated with $Q$ replacing $M$ then we can conclude that $Q$ is indecomposable.

One might ask if the existence of a maximal ordered subset $L$ of $E(M)$ whose closure contains the boundary of $M$ is equivalent to the boundary of $M$ being indecomposable. The answer is no. However, the following holds.

(1) If the boundary of $M$ is indecomposable then for each simple closed curve $D$ such that $D \cap M$ is finite and $M \subset T(D)$, there is a component $K$ of $D-M$ for which $[T(K \cup M) \cap E(M)]^{*}$ contains the boundary of $M$.

(2) If for each simple closed curve $D$ such that $D \cap M$ is finite and $M \subset T(D)$ there is a component $K$ of $D-M$ such that $[T(K \cup M) \cap E(M)]^{*}$ contains the boundary of $M$, then the boundary of $M$ is indecomposable or is the union of exactly two indecomposable continua.

It is easy to construct an example of an indecomposable continuum $M$ for which (1) holds where $L^{*}-L$ has exactly one point for any maximal ordered subset of $E(M)$. 


\section{BIBLIOGRAPHY}

1. Samuel Eilenberg and Deane Montgomery, Fixed point theorems for multi-valued transformations, Amer. J. Math. 68 (1946), 214-222.

2. Shizuo Kakutani, A generalization of Brouwer's fixed point theorem, Duke Math. J. 8 (1941), 457-459.

3. A. D. Wallace, Classroom notes for algebraic topology, Tulane Univ., New Orleans, La.

4. G. Hocking and G. S. Young, Topology, Addison-Wesley, Reading, Mass., 1956.

State University OF New York,

Stony BrooK, L. I., New York 